\title{
Difficultés des canaux de distribution alimentaire en territoire rural : l'importance du leader
}

The challenges of sustainable distribution channels in rural areas: The importance of the leader

\section{Virginie Noireaux et Patrick Ralet}

\section{(2) OpenEdition}

\section{Journals}

Édition électronique

URL : http://journals.openedition.org/economierurale/6918

DOI : 10.4000/economierurale.6918

ISSN : 2105-2581

Éditeur

Société Française d'Économie Rurale (SFER)

Édition imprimée

Date de publication : 30 septembre 2019

Pagination : 21-39

ISSN : 0013-0559

Référence électronique

Virginie Noireaux et Patrick Ralet, « Difficultés des canaux de distribution alimentaire en territoire rural : l'importance du leader », Économie rurale [En ligne], 369 | Juillet-septembre, mis en ligne le 01 janvier 2021, consulté le 08 janvier 2021. URL : http://journals.openedition.org/economierurale/6918 ; DOI : https://doi.org/10.4000/economierurale.6918 


\title{
Difficultés des canaux de distribution alimentaire en territoire rural : l'importance du leader
}

\author{
Virginie NOIREAUX • IAE d'Auvergne Clermont Université, Centre de Recherches Clermontois en \\ Gestion et Management (CRCGM) EA3849, Clermont-Ferrand \\ virginie.noireaux@uca.fr \\ Patrick RALET • IAE d'Auvergne Clermont Université, Centre de Recherches Clermontois en \\ Gestion et Management (CRCGM) EA3849, Clermont-Ferrand \\ patrick.ralet@uca.fr
}

L'objectif de cet article est d'expliquer les freins existants à la mise en place de solutions efficientes et durables dans les canaux de distribution alimentaire en contexte rural, en se concentrant sur le rôle joué par les leaders de ces canaux. L'analyse de CapLog, groupe de travail réunissant des acteurs de la distribution du Massif central, a permis d'analyser de manière qualitative et exploratoire les attributs du leader. Les membres ont une vision convergente du canal et ont acquis une expertise marketing. Mais le manque d'expertise logistique et l'absence d'un leader légitime restent un frein à la pérennité des solutions proposées. Les auteurs concluent sur les compétences que devraient acquérir les acteurs pour se positionner comme de véritables leaders de canaux de distribution compétitifs.

MOTS-CLÉS : distribution alimentaire, leader, ruralité, organisation alimentaire

\section{The challenges of sustainable distribution channels in rural areas: The importance of the leader}

The objective of our work is to explain the existing obstacles to the implementation of efficient and sustainable solutions in food distribution channels for remote rural areas, focusing on the role played by the leaders of these channels. The analysis of CapLog, a working group of distribution actors in the Massif Central, has enabled us to analyze in a qualitative and exploratory way the attributes of the leader. Although the members share the vision of the channel and have acquired marketing expertise, a lack of logistical expertise and the absence of a legitimate leader remain obstacles to the sustainability of the solutions proposed. We conclude on the skills that actors should acquire in order to position themselves as true leaders of competitive distribution channels. (JEL: MO)

KEYWORDS: food distribution, leader, rural areas, food organization

L es évolutions des canaux de distribution tendent à évoluer vers une dimension plus locale : les relations des acteurs s'inscrivent sur un territoire « à taille humaine », qui correspond à une origine géographique clairement identifiée (Merle et Piotrowski, 2012). Les débats politiques font d'ailleurs écho à cette tendance, puisque le document de la politique alimentaire nationale de 2015 fait référence à
« l'ancrage territorial pour soutenir le modèle agricole français en favorisant l'approvisionnement de proximité ou d'origine française, et en s'appuyant sur des platesformes permettant de faire se rencontrer offre et demande $»^{1}$.

1. https://www.gouvernement.fr/action/unenouvelle-politique-de-l-alimentation 
Si l'évolution de la distribution est largement abordée dans la littérature sous l'angle des consommateurs citadins (Cara et al., 2017 ; Rose et al., 2017), les recherches abordant spécifiquement les questions de distribution dans les zones rurales sont moins répandues. Or la ruralité engendre des problématiques de distribution particulières. Alors que, sur un territoire urbain, la massification des flux à l'entrée des villes domine (Rose et al., 2017), en milieu rural, leur dispersion engendre de nombreuses conséquences sur les stratégies de distribution. Ainsi, l'évolution de la demande et de la consommation incite les acteurs à innover en termes de distribution.

Dans les territoires urbains, cette innovation est généralement portée par les acteurs majeurs du secteur (grands distributeurs et acteurs du commerce en ligne) au sein de systèmes marketing verticaux déjà existants. Ces derniers sont définis comme des organisations stables qui coordonnent le processus d'intermédiation entre producteur et acheteur (McCammon, 1970). Dans les zones rurales, les projets de systèmes marketing verticaux existent, mais sont plutôt portés par des acteurs locaux. C'est à eux que nous nous intéressons. Nous avons suivi pendant près de cinq années le groupe de travail CapLog dont l'objectif est d'identifier les meilleures pratiques ou innovations de distribution dans le Massif central. Or il apparaît une véritable difficulté de coordination des opérations et des ressources dans ces canaux. La coordination est pourtant essentielle, car elle permet aux membres de bénéficier d'économies importantes et de maximiser la qualité des prestations offertes aux clients (Christopher, 2016). Ce rôle, dans les canaux de distribution, est assumé par le leader (Noireaux et Poirel, 2009), qui distribue les tâches à l'ensemble des membres et coordonne les opérations, afin de garantir la compétitivité du canal. L'objectif de notre travail est d'expliquer les freins existants au fonctionnement ou à la mise en place de canaux de distribution alimentaire en milieu rural, à travers les défauts de coordination du leader. En effet, nous déduisons que les leaders des canaux de distribution en milieu rural rencontrent des difficultés à développer les attributs essentiels au bon fonctionnement du canal. Pour ce faire, nous présentons la distribution en contexte rural, ainsi que le rôle et les attributs que doit posséder le leader. Nous exposons ensuite, à travers l'étude du groupe de travail CapLog, les freins liés à l'émergence d'un leader garant de solutions efficientes.

\section{Les évolutions de la distribution et la place de la ruralité}

Une étude prospective sur les comportements alimentaires en 2025 souligne l'importance croissante d'une alimentation pérenne et soucieuse de bien-être (Blézat et al., 2017). Au-delà du souci d'utiliser moins d'emballages ou de promouvoir des produits respectueux de l'environnement, cette notion s'appuie sur une volonté de repenser les relations entre les acteurs. Il s'agit par exemple de nouvelles pratiques de consommation collaborative au service d'une alimentation plus durable. Que ce soit via l'application Partagetonfrigo ou la plate-forme La Ruche qui dit oui !, la tendance est de changer les comportements, en mettant en valeur une plus grande proximité entre producteurs et consommateurs.

Plus largement, en matière alimentaire, un lien direct est établi entre développement à moyen terme et proximité (Blézat et al., 2017). La volonté de promouvoir des systèmes équitables et locaux se traduit par le développement de circuits courts et de proximité. Au-delà de la juste rémunération des producteurs, l'objectif est de mettre en place des accords qui renforcent les compétences organisationnelles et techniques des acteurs. Les travaux réalisés sur l'analyse des Systèmes agroalimentaires 
localisés (SYAL) ont également souligné depuis plusieurs années l'importance de s'inscrire dans un contexte territorial donné pour mettre en place des solutions de développement (Muchnik et al., 2007 ; Cerdan et al., 2017). Le développement des canaux de proximité répond alors à une double attente du consommateur. En premier lieu, un souhait de garantie sur la qualité de ce qu'il ingère (Moati, 2018) à travers des produits « anciens », « non calibrés » ou «naturels ». En second lieu, une recherche d'identité (Delavigne, 2001) via les produits alimentaires, qui deviennent un vecteur privilégié de distinction et d'affirmation d'une identité personnelle et communautaire.

Reste que l'évolution des canaux ne saurait être sans conséquence sur l'ensemble des acteurs de la distribution (Moati, 2018). De l'amont à l'aval, chaque acteur de la distribution est interrogé sur ses pratiques et sa capacité à saisir les opportunités potentielles de développement.

Les agriculteurs décèlent dans une demande plus qualitative de nouvelles possibilités de développer leur activité, sous réserve de satisfaire aux exigences croissantes de transparence et de maîtriser leur impact global au niveau environnemental (Duru et al., 2017).

Les transformateurs cherchent à intégrer les conséquences d'une individualisation des modes et des rythmes de vie en matière alimentaire (Gontard et al., 2017). La variété des portions (de l'individuel au familial), les modes de consommation (faire soi-même ou prêt à consommer, repas traditionnel ou grignotage, chez soi ou à l'extérieur) ou les produits euxmêmes (« expériences » culinaires...) ont une incidence directe sur les logiques de stockage, de préparation et de conditionnement.

Le développement d'une activité locale est ensuite une opportunité pour les transporteurs et les logisticiens (Vaillant et al., 2017), mais il implique de maîtriser certaines données de base telles que la collecte, l'organisation des tournées et le stockage, avec leurs coûts associés. La désaffection relative des grandes surfaces par les consommateurs traduit d'ailleurs bien les changements en cours. Le client final veut pouvoir choisir. Aussi doit-il se comporter parfois en véritable stratège, recherchant avec l'aide des comparateurs de prix et des réseaux sociaux, le meilleur rapport qualité/prix (Blézat et al., 2017 ; Moati, 2018).

Pourtant, malgré l'intérêt soulevé par la distribution en milieu rural, peu de solutions originales sont mises en place. Généralement, c'est la déclinaison de solutions existantes en milieu urbain qui domine. La grande distribution a bien souvent étendu au milieu rural - sous contraintes de rentabilité - des organisations ayant fait leurs preuves en milieu urbain. De plus, le consommateur rural doit généralement développer un effort de mobilité supplémentaire pour avoir accès à une offre souvent plus limitée en nombre et en diversité (Vaillant et al., 2017). L'achat en ligne n'a fait de ce point de vue que déplacer le problème des coûts d'accès vers les acteurs de la livraison.

\section{Un leader nécessaire au bon fonctionnement des canaux de distribution}

La littérature souligne l'importance centrale de la coordination des acteurs dans les systèmes agroalimentaires locaux (Vázquez, 2005). Cette coordination est traduite par la notion de pilotage inhérente au canal de distribution (Mehta et al., 1996 ; Christopher, 2016), et qui se traduit par une volonté d'intégration des processus des différents éléments du canal (Guérin et Lambert, 2012). Dans les canaux, le pilotage est assuré par le leader, représenté par une organisation qui distribue les tâches au sein du canal et fixe les objectifs (Mallen, 
1964 ; Stern et El-Ansary, 1988 ; White et Bednar, 1991). L'inexistence d'un leader conduit à l'anarchie, où chacun décide seul et sans entrave, et mène à la destruction du canal (Mallen, 1964).

Dès le départ, les auteurs (Mallen, 1964 ; Stern et El-Ansary, 1988) cherchent à analyser les différents leaders possibles dans le canal. Ils concluent que n'importe quel membre peut en devenir le leader. Il faut également que l'acteur veuille bien endosser ce rôle, ce qui n'est pas toujours le cas (Stern et El-Ansary, 1988 ; White et Bednar, 1991).

Dans les analyses faites dans la littérature, le leader est représenté par :

- Soit l'acteur qui possède le pouvoir prédominant dans la chaîne (Perotti et al., 2012).

- Soit l'acteur qui développe une capacité supérieure à coordonner l'ensemble des acteurs (Christopher, 2016). Cette dernière hypothèse est notamment développée par les travaux qui soulignent le rôle du prestataire de services logistiques (Fabbe-Costes et Roussat, 2011 ; Chanut et Paché, 2013).

- Soit un réseau coopétitif (Le Roy et al., 2008) qui associe des concurrents adoptant des stratégies coopératives.

- Soit une stratégie collective (Noireaux et Poirel, 2014, 2017), qui regroupe différentes parties prenantes privées et publiques afin d'organiser le canal.

La place de l'institution leader a été largement débattue dans la littérature, mais elle n'a pas donné de résultat formel. Seuls les consommateurs ne sont pas considérés comme pouvant endosser ce rôle (Stern et El Anasary, 1988).

\section{Les attributs du leader pour un canal fonctionnel}

Si l'existence du leader est inhérente à celle des canaux de distribution, le leadership a un caractère dynamique. L'adaptation des canaux de distribution à une nouvelle demande ou encore la création de nouveaux canaux représentent des perturbations stratégiques pour le leader qui sont susceptibles d'affaiblir ou d'accroître son rôle de coordination, et même de lui faire perdre son leadership au profit d'un autre acteur (Stern et El Anasary, 1988).

Le leader doit posséder et développer certains attributs. Ces attributs doivent être reconnus par les autres membres du canal. Ils permettent au leader d'éviter les conflits dysfonctionnels, ceux qui mettent en péril l'atteinte des objectifs et/ou le canal luimême. Si les conflits sont inhérents au canal, les auteurs distinguent les conflits fonctionnels des conflits dysfonctionnels. Les conflits fonctionnels représentent une tension entre les membres, mais ils n'affectent pas l'atteinte des objectifs ni la viabilité du canal. Ils sont même utiles car vecteur d'innovation (Stern et El-Ansary, 1988). Les conflits dysfonctionnels réduisent la performance et l'efficience du canal (Stern et El-Ansary, 1988). Ils peuvent même conduire à sa destruction (Noireaux et Poirel, 2009).

Pour assurer une bonne coordination et éviter les conflits dysfonctionnels, le leader doit revêtir trois attributs (Noireaux et Poirel, 2009). Le premier correspond à la vision du leader qui doit être proche ou concordante avec celle des autres membres. Si c'est le cas, ils cherchent alors à collaborer dans le canal et aident à atteindre les objectifs. Cet attribut est également souligné par Teng et Thomas (2017), pour qui le premier composant lorsque l'on configure un canal est la création de valeur, qui doit être partagée par les clients et par les membres. Pour Gattorna et Tang (2003), la vision représente la manière dont les acteurs se positionnent par rapport à la question «que voulons-nous défendre? ». La vision de la future organisation doit représenter pour chacun des bénéfices qui peuvent être entendus sous forme de valeur 
monétaire ou qualitative (Tang et Thomas, 2017 ; Gattorna et Tang, 2003). De nombreux échecs de construction de canaux s'expliquent par une absence de bénéfices escomptés par un ou plusieurs acteurs. C'est donc le premier élément à déterminer (Gattorna et Tang, 2003).

Le second attribut est lié à l'expertise du leader et à sa connaissance de l'environnement. Sa fonction est d'organiser et de coordonner au mieux le travail en fonction des objectifs (vision) qu'il impose. Cette fonction est déterminée par ses compétences et son expertise. En distribution, l'expertise porte sur deux dimensions, qualifiées de «deux moitiés de la distribution » : la logistique et le marketing, qui permettent une meilleure articulation de l'offre et de la demande (Converse, 1958). L'expertise porte à la fois sur les aspects techniques du canal, mais également sur les aspects organisationnels. Pour Tang et Thomas (2017), il s'agit de définir la composition des membres du canal, de les décrire techniquement, puis d'identifier le volume des flux. Ainsi, une bonne connaissance de l'environnement permettra de savoir à qui il faut laisser une certaine autonomie, et à qui il faut confier certaines tâches. La connaissance technique permettra de savoir quelles sont les tâches à réaliser, comment et à quel coût.

Enfin, le dernier attribut est lié à la légitimité du leader. Celle-ci peut être perçue à deux niveaux. Le premier représente la légitimité du leader aux yeux des autres membres. S'il est considéré comme légitime, ses décisions, l'utilisation de son pouvoir et sa vision seront mieux perçues par les membres. Sa légitimité peut donc être renforcée par les deux attributs précédemment cités, à savoir la vision et l'expertise. Il peut ainsi apparaître légitime aux yeux d'un acteur parce qu'il poursuit le même objectif, ou parce qu'il possède des compétences que l'acteur ne possède pas. Il peut également être légitime du fait de sa place dans le canal ou du fait de l'historique des relations (Noireaux et Poirel, 2009). Cette conception se rapproche de celle développée par Stern et El-Ansary (1988) qui préconisent d'analyser le niveau de tolérance de chaque membre visà-vis du contrôle par des leaders potentiels, afin de déterminer le membre le mieux à même de tenir le rôle de leader.

Le second niveau de légitimité est celui de l'acteur lui-même. Les membres du canal ne cherchent pas forcément à devenir leader, car cela dépend de leurs espoirs de performance (Anand et Stern, 1985). Cette perception peut être objective ou subjective et peut être le résultat d'une situation actuelle ou bien basée sur l'analyse d'une situation hypothétique (Anand et Stern, 1985). Nous retiendrons donc ces trois attributs de vision, d'expertise et de légitimité, afin d'analyser les difficultés des canaux de distribution des produits locaux dans les territoires ruraux.

\section{Méthodologie}

L'analyse des attributs du leader de canal de distribution alimentaire en contexte rural nécessite l'utilisation d'une méthode qualitative pour comprendre les enjeux réels et la conduite des acteurs en interaction ou en situation de dépendance (Baumard et Ibert, 2007). L'approche qualitative, même si elle est peu employée dans le courant behavioriste, permet de produire une compréhension fine des processus et de dégager des éléments théoriques pertinents (Hunt, 2015). Notre approche par étude de cas se justifie également par la complexité du problème à étudier et par le fait que l'étude rentre dans un champ relativement nouveau (Hlady-Rispal, 2015).

Le cas étudié est celui du groupe de travail CapLog que nous avons suivi pendant près de cinq ans. Ce groupe de réflexion, composé de différents acteurs privés et publics, s'intéresse à la distribution 
alimentaire durable dans les territoires de moyenne montagne du Massif central.

La collecte des données s'est faite de 2013 à 2018 :

- par la participation à 16 réunions trimestrielles du groupe de travail de $3 \mathrm{~h}$ à $4,5 \mathrm{~h}$, à une réunion spécifique regroupant 15 agriculteurs,

- par la réalisation de 15 entretiens individuels (d'une durée variant de $45 \mathrm{mi}$ nutes à $2 \mathrm{~h} 50$ ) avec des membres du groupe de travail : 5 associations (2 Associations pour le maintien de l'agriculture paysanne [AMAP], 2 associations aidant les acteurs à mettre en place le développement durable et 1 'association de producteurs), 5 entreprises (3 distributeurs: un drive, un magasin de producteur, un grand distributeur-la personne interrogée est également présidente de la Fédération de la distribution et du commerce
[FDC] -, 2 grossistes) et 5 institutions (Département du Cantal, Saint-Flour Communauté, Communauté d'agglomération de Vichy, Chambre d'Agriculture Auvergne Rhône-Alpes et Chambre de métiers et de l'artisanat Auvergne).

Les entretiens individuels et la réunion spécifique avec des agriculteurs nous ont donc permis de limiter le biais relatif au recueil de données en réunion collective, où certains aspects peuvent être minimisés ou davantage soulignés du fait de la présence et de l'interdépendance avec d'autres parties prenantes. Le choix des personnes interrogées s'est fait en fonction de leur expertise sur une catégorie d'acteurs (Union régionale des industries agro-alimentaires de l'Auvergne [URIAA] pour les transformateurs, FDC pour la grande distribution, chambre régionale d'agriculture pour les agriculteurs...), sur un type de distribution spécifique (restauration collective, drive,

\section{Présentation du cas CapLog}

Le groupe de travail CapLog a été créé à la suite du colloque professionnel « Circuits alimentaires de proximité : quelles coordinations entre acteurs pour développer les circuits alimentaires de proximité en Massif central ? », organisé le 22 novembre 2012 à Saint-Flour. Ce colloque a mobilisé plus de 150 professionnels. À l'issue du colloque, CapLog a émergé après l'analyse des insuffisances recensées et validées par les acteurs lors des débats. CapLog est un groupe de travail qui s'intéresse à la thématique suivante: «circuits alimentaires de proximité : quelle logistique pour quels marchés pour un développement durable en milieu rural de moyenne montagne?».

Le groupe de travail se compose de 35 organisations privées et publiques (annexe 1) engagées de manière active (présence aux réunions au moins une fois par an, transmission de documents et retours d'expériences). Chaque membre du groupe de travail est une partie prenante d'un ou plusieurs canaux de distribution sur le territoire étudié. L'objectif de ce groupe de travail est de comprendre et d'accompagner les canaux de distribution alimentaires dans un contexte spécifique de territoire rural. Les acteurs se regroupent dans le but d'échanger sur les pratiques existantes et sur les projets de nouveaux canaux en cours de construction, afin de trouver des solutions efficientes, permettant éventuellement leur duplication sur le territoire.

L'objectif de Caplog n'est pas de mettre en place une stratégie de distribution spécifique sur le territoire, mais d'identifier les différentes pratiques de distribution des acteurs en milieu rural et de comprendre les raisons des succès ou échecs observés. En parallèle des réunions de CapLog, nous avons réalisé des entretiens avec des acteurs spécifiques du groupe, afin de mieux comprendre certains aspects étudiés ou d'approfondir certaines difficultés. 
RECHERCHE

AMAP, commerce indépendant et intégré), sur certaines spécificités territoriales (Pays de Vichy Auvergne, Saint-Flour Communauté, Réseau rural Auvergne, Conseil général) ou sur l'accompagnement de projets innovants (MACEO, PF21 pour le développement durable). Le traitement des données a été réalisé à travers l'élaboration de comptes rendus de réunion et de fiches de synthèse d'entretiens. Nous avons complété ces données par l'analyse de documents fournis par les acteurs (notamment des études locales et nationales), qui nous ont permis d'avoir accès à des données sur les attentes des consommateurs. Les éléments du cas et des entretiens concernent le cas spécifique du Massif central. Pour traiter les données, nous avons distingué les principaux canaux identifiés dans CapLog et leurs leaders (actuels ou potentiels).

La participation au groupe de travail CapLog nous a permis d'identifier différentes formes de distribution existantes sur le territoire (tableau 1). Le tableau 1 présente l'ensemble des canaux d'alimentation identifiés sur le territoire par les acteurs. Chaque canal est illustré par un exemple anonyme détaillé dans les tableaux 2 et 3. Certains canaux sont plus développés que d'autres (comme la grande distribution ou les AMAP). Le tableau 1 distingue les canaux existants et les canaux en construction. Nous avons retenu uniquement les canaux intégrés qui visent l'intégration des flux à travers la centralisation des décisions par un acteur ou un groupe d'acteurs. Ceci nous a conduits à ne pas retenir certaines formes de distribution sur le territoire, comme les marchés ou la vente à la ferme. En effet, la structure de ces canaux est considérée comme traditionnelle du fait de l'absence de formalisation des relations entre les membres (McCammon, 1970).

\section{Tableau 1. Les formes de distribution identifiées dans la Massif central}

\begin{tabular}{|c|c|c|}
\hline Forme du canal & Développement de la forme sur le territoire & Exemple $n^{\circ}$ \\
\hline Drive fermier & Peu développé (moins de 10 canaux) & 1 \\
\hline $\begin{array}{l}\text { Vente en ligne via une plate-forme de } \\
\text { regroupement }\end{array}$ & Très peu développé (moins de 3 canaux) & 2 \\
\hline Grande Distribution & Fortement développée (plus de 50 canaux) & 3 \\
\hline AMAP & Fortement développée (plus de 50 canaux) & 4 \\
\hline Magasins indépendants (petite taille) & Développé mais chiffrage NC & 5 \\
\hline $\begin{array}{l}\text { Regroupement et distribution via une } \\
\text { plate-forme locale }\end{array}$ & Très peu développé (moins de 3 canaux) & 6 \\
\hline $\begin{array}{l}\text { Optimisation des flux vendus par une } \\
\text { plate-forme d'achat existante (restauration } \\
\text { collective) }\end{array}$ & Une par département & 7 \\
\hline
\end{tabular}




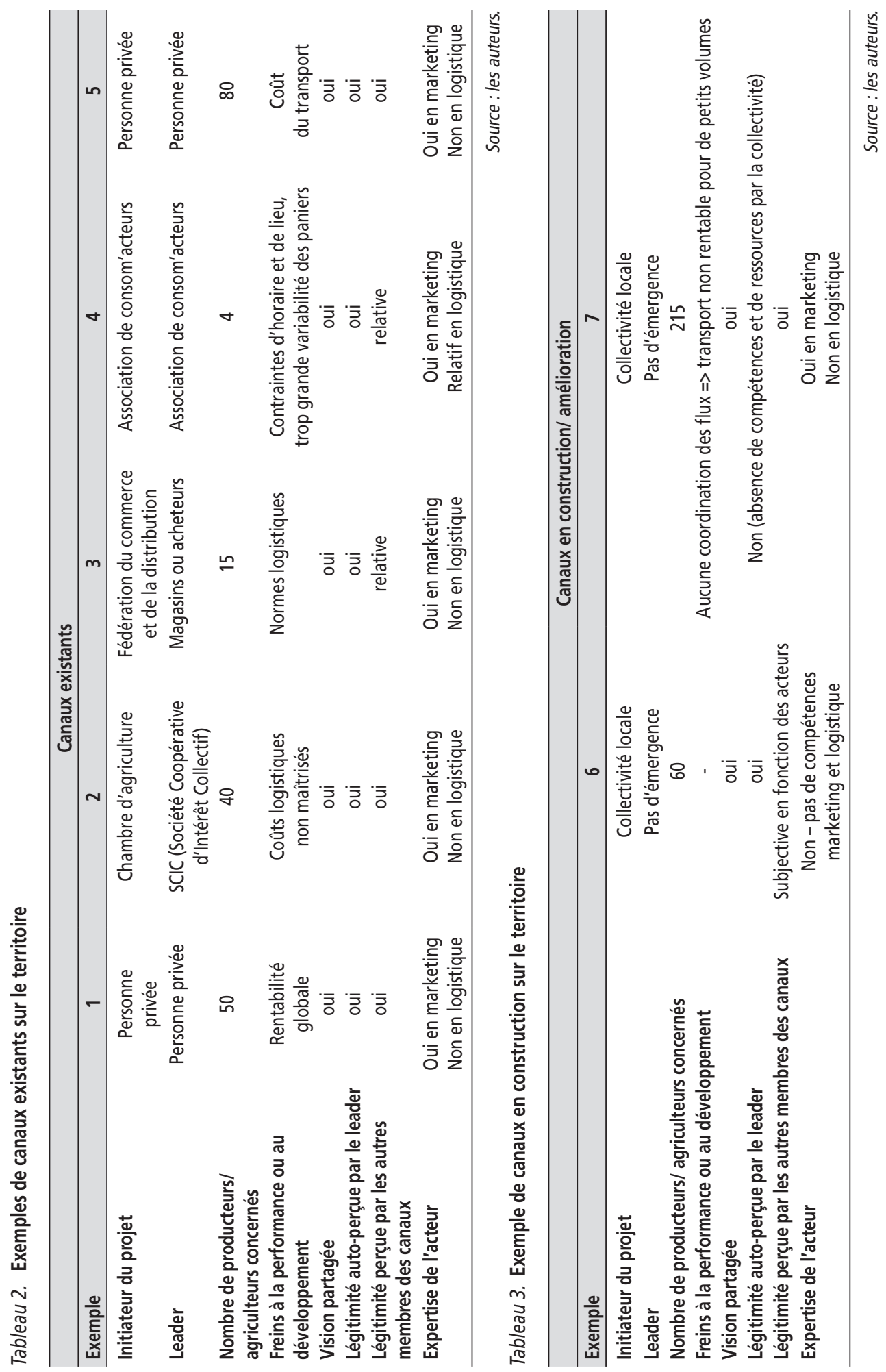




\section{Résultats}

Nous présentons les résultats du groupe de travail et des entretiens, en abordant successivement les trois attributs essentiels attendus d'un leader pour créer un canal fonctionnel : la vision, la légitimité et l'expertise.

\section{Une vision convergente des acteurs sur la finalité}

La vision du canal, c'est-à-dire la formulation des bénéfices attendus du développement de canaux de distribution alimentaire en milieu rural, montre une forte convergence entre les acteurs (tableau 4). Les bénéfices escomptés ont été déterminés par les acteurs eux-mêmes et représentent les gains que ces derniers pourraient obtenir à travers le développement des canaux.

En suivant les acteurs de l'amont vers l'aval, nous observons que certains producteurs agricoles, ancrés au sein de territoires très retirés, se retrouvent face à des difficultés liées aux «premiers kilomètres ».
Le premier kilomètre représente le coût du premier maillon de la chaîne logistique partant du producteur et à destination d'un autre acteur du canal (intermédiaire ou consommateur): temps de transport plus important sur les routes secondaires, déplacement pouvant devenir aléatoire en moyenne montagne (enneigement, gel, congestions des routes touristiques à certaines périodes), prix de transport plus important du fait de l'écartement des grands axes, difficultés de trouver un transporteur qui se déplace. Ces freins impactent non seulement les coûts, mais aussi la transmission des exploitations, qui devient alors plus problématique.

«La transmission des exploitations est un point critique ici. Dans les cinq à dix ans à venir, plus de la moitié des exploitations devront être transmises. Il faut assurer la rentabilité, mais également la qualité de vie de l'agriculteur...» (Chambre d'agriculture, compte rendu de réunion, $08 / 11 / 2016$ )

\section{Tableau 4. Vision déterminée par les acteurs}

\begin{tabular}{ll}
\hline Acteurs & \multicolumn{1}{c}{ Enjeux pour l'acteur } \\
\hline Agriculteurs & - Augmenter la rentabilité \\
& - Faire évoluer la production en fonction de la demande (quantité / type de \\
& produits / qualité) \\
& - Gérer la logistique du premier kilomètre \\
& - Transmettre les exploitations \\
Industries de & - Diminuer le coût des transports de la distribution locale \\
transformation & - Développer des compétences et ressources liées à la supply chain locale \\
& - Investir (ou pas) dans les normes sectorielles imposées par les supply chains \\
& de la grande distribution \\
Prestataires logistiques & - Mutualiser des flux surtout dans les territoires excentrés \\
Grossistes locaux & - Adapter leurs activités pour survivre/ se développer \\
Distributeurs & - Mailler suffisamment le territoire \\
Consommateurs & - Rentabiliser les points de vente \\
Institutions & - Faire des achats un acte symbolique et/ou écologique \\
& - Garder les services publics et privés même dans les zones rurales \\
& - Maintenir ou augmenter la population sur certains territoires (attractivité \\
& économique et touristique) \\
\hline & - Maîtriser le coût du service public et des investissements
\end{tabular}


Le développement de canaux semble, selon eux, pouvoir répondre en partie à ces difficultés en regroupant les flux et en minimisant le temps consacré aux tâches logistiques.

Plus en aval, au niveau des industries de transformation, d'autres bénéfices émergent comme la promotion et la distribution de produits locaux, et plus spécifiquement la rentabilité des transports de petits volumes.

«Certains adhérents sont voisins et livrent dans les mêmes villes, et parfois dans les mêmes commerces... Seulement l'information ne passe pas et ils payent "plein pot". » (URIAA, 22/11/2013)

À un autre niveau, les entreprises logistiques se posent la question de la mutualisation des flux (regroupement /éclatement) dans le but de parvenir à des charges complètes. Pour les grossistes, ces canaux représentent un moyen de survie ou de développement.

«Si je peux me développer avec cette distribution, je prends! La conjoncture est très difficile... » (Grossiste, 25/06/2013)

Enfin, la grande distribution s'intéresse également à l'approvisionnement en produits locaux. En effet, la demande pour ces derniers est croissante, mais les cahiers des charges pour les producteurs sont contraignants en matière d'emballages, de palettisation ou de volumes demandés... Or beaucoup de producteurs locaux ne sont pas en mesure de répondre à ces exigences, même lorsque les distributeurs commandent directement aux producteurs (certains des chefs de rayon bénéficiant d'une autonomie locale d'approvisionnement).

«Nous cherchons à développer l'offre locale. Dans le groupe (Carrefour), je participe à la cellule PROXI qui réfléchit à l'approvisionnement local. » (FCD, Gérant Carrefour, 10/10/2014)
Les formes de distribution et leur adaptation au contexte local sont également un objectif pour les distributeurs dans un contexte de disparition de certains commerces sur certaines zones du territoire. Les consommateurs souhaitent être informés plus précisément de la provenance des produits (région, village...), accéder à une grande diversité de produits alimentaires et aux services publics et privés le plus près possible de chez eux.

«Les consommateurs font de plus en plus confiance aux produits agricoles de proximité. » (Ipsos, 2014)

Les acteurs institutionnels examinent deux aspects spécifiques. Le premier tient au développement et/ou au maintien de l'attractivité du territoire pour les acteurs économiques (reprises et créations de filières, aide au maintien de la distribution, attractivité liée au tourisme, notamment au tourisme « vert »). Le second aspect est lié à la maîtrise du coût des services publics (cantine, maison de retraite...) ou des investissements (routes...) dans un contexte où les recettes locales de l'activité économique et les dotations étatiques sont de plus en plus limitées.

Dans le Massif central, la vision de ces canaux semble donc largement convergente en termes de buts à atteindre. Et les problématiques logistiques apparaissent centrales, de ce point de vue, pour la majorité des membres. Cependant, à l'échelle locale, le développement ou la continuité des actions logistiques réalisées restent limités.

\section{Une légitimité pas toujours partagée}

Face à une vision somme toute convergente des acteurs sur le type de canal qu'ils souhaitent défendre, émerge-t-il pour autant un leader doté de la légitimité nécessaire? La position des différents acteurs est contrastée.

Les agriculteurs, les transformateurs et certains distributeurs indépendants 
RECHERCHE

Virginie NOIREAUX, Patrick RALET

souhaitent se concentrer sur l'offre de produits. Ils peuvent, dans le cadre de contrats, acheminer les produits chez un client ou bien aller en chercher chez un fournisseur, en assurant le déplacement eux-mêmes ou en faisant appel à un transporteur. Toutefois, ils ne cherchent pas réellement à optimiser le transport.

«J'ai un camion qui fait les tournées habituelles et j'utilise aussi Chronofresh ou Transprim. Mon chauffeur fait les livraisons au jour le jour en fonction des demandes. » (GAEC production et transformation porcine, 13/09/2018)

Que la logistique soit internalisée ou sous-traitée à un transporteur, ils estiment que l'organisation des flux dans le canal de distribution ne relève pas de leur cœur de métier. Ils sont toutefois parfois prêts à adapter leurs produits et leurs emballages en fonction de la demande d'un éventuel leader (sous réserve de capacité d'investissement), en répondant à ses normes (palettisation des produits...).

La grande distribution pour sa part se positionne naturellement comme leader. Cependant, nous avons constaté dans le cadre de notre étude qu'une partie des producteurs refuse son leadership pour des raisons soit financières :

«Je me refuse de vendre à perte.» (agriculteur parlant de la grande distribution, 26/07/2018),

soit éthiques :

«J'adhère à la mention "nature et progrès” qui ne correspond pas à la grande distribution. » (Agriculteur),

soit de dépendance commerciale :

«Je ne peux pas m'engager pour les volumes demandés, sous peine d'arrêter les autres modes de distribution 》 (agriculteur, 13/09/2018).

Les prestataires et les grossistes peuvent répondre à des demandes simples ou complexes de transport et de logistique :
«Il précise qu'il peut répondre à toutes les demandes, la logistique c'est son métier. Mais il n'est pas commerçant...» (Prestataire, compte rendu de réunion, 23/05/2015)

Mais pour la plupart, ils ne souhaitent pas endosser le rôle de leader en milieu rural, notamment car le marché leur paraît peu rentable à court terme. Dans le cadre de CapLog, certains grossistes, notamment ceux en difficulté, sont, en revanche, prêts à tenir ce rôle, pour assurer leur survie.

« Je suis en attente de solutions... ditesmoi comment faire, car mon activité ne peut plus continuer ainsi. » (Grossiste, 25/06/2013)

Les consommateurs, dans leur majorité, ne souhaitent pas organiser leur approvisionnement, même si certains d'entre eux, notamment dans le cadre d'organisation de type AMAP, peuvent s'approprier ce rôle. Les enquêtes sur les pratiques en la matière montrent que le consommateur «ne parcourt pas plus de $10 \mathrm{~km}$ pour l'achat de ses produits locaux; il est freiné par le prix et la distribution de ces produits » (IPSOS, 2014).

Et les consommateurs ne sont pas toujours tenus pour légitimes par les autres acteurs. C'est le cas dans une AMAP où certains producteurs se sentent dépossédés de certaines tâches liées à leur métier comme le choix des semences.

"C'est très difficile de discuter avec eux [association de consommateurs]. Ils ont des idées très précises sur ce que je dois faire et quand... et moi j'exécute. Ce n'est pas le métier que j'ai choisi...» (Producteur, AMAP, 2/10/2015)

Les institutions sont également en difficulté pour accéder à ce rôle. En effet, elles ont pour vocation de fournir aux populations un service public de qualité. Bien que les collectivités perçoivent souvent clairement l'intérêt général et soient prêtes à 
aider des actions locales par des études, des financements ou de l'aide à l'installation, elles ne possèdent pas ou peu de ressources et de compétences en matière de distribution alimentaire et pensent ne pas être légitimes pour assurer ce rôle. Ce qui est d'ailleurs partagé par certains autres acteurs :

«... elle ne peut pas tout faire. Est-ce le rôle du service public de faire de la distribution alimentaire? - Remarque : approbation des autres membres...» (Conseil général, compte rendu réunion, 8/11/2016)

\section{L'expertise : la véritable difficulté}

Le frein principal aux canaux de distribution en contexte rural semble provenir du manque de compétences logistiques. D'après les acteurs, si les compétences marketing semblent acquises, les compétences logistiques sont un véritable facteur limitant.

Les agriculteurs, les industries locales de transformation, les collectivités locales et les consommateurs ne possèdent pas les compétences nécessaires pour organiser la logistique de distribution. Cela s'explique du fait de leur cœur de métier ou de leur rôle dans l'histoire du canal. Les leaders des cas 1 et 2 reconnaissent cette difficulté. Dans les deux cas, l'expertise marketing a été privilégiée à l'expertise logistique.

"Sur la plate-forme nous avons deux employés qui sortent de l'option Marketing de [École]... Nous nous sommes rendu compte au fur et à mesure de la difficulté d'organiser les transports, de gérer les stocks. » (Chambre d'agriculture, compte rendu de réunion, 3/06/2016)

«J'ai pris conscience de la difficulté du Drive... je ne m'attendais pas à une telle complexité... » (Gérant Drive, 05/10/2017)

Dans le cadre des AMAP, certaines tâches logistiques sont organisées par l'association (préparation des paniers en amont, livraison au point de réception...).
Cependant, la récupération des paniers se fait à date et heure fixe, ce qui peut représenter un frein pour certains consommateurs.

«Le développement des AMAP est limité par la rigidité que cela représente : date et heure fixe, diversité... » (Conseil général, compte rendu de réunion, 2/10/2015)

Les grossistes locaux et les prestataires logistiques pourraient apporter une contribution importante en développant des ressources et des compétences adaptées. Mais, dans le contexte du Massif central, certains grossistes sont très affaiblis financièrement, ce qui ne leur permet pas de déployer des ressources.

«Je suis très mal... je ne peux pas investir. »(Grossiste, 8/11/2016)

D'autres possèdent davantage d'activités mais ne se sentent pas légitimes pour tenir ce rôle. C'est également le cas des prestataires qui ne développent pas de ressources ou de compétences spécifiques, tant qu'il n'y a pas de demande précise.

Les acteurs de la grande distribution sont limités par l'offre de produits apportée par les centrales d'achat. Dans certains groupes, les chefs de magasin ou de rayon peuvent bénéficier d'une certaine autonomie d'approvisionnement sur le marché local, mais dans ce cas, ils n'organisent pas la logistique qui reste à la charge du fournisseur pour tout (livraison directe) ou partie (livraison à l'entrepôt local) du flux.

«La logistique reste à la charge des producteurs locaux dans la grande majorité des contrats. » (FCD, compte rendu de réunion, 23/05/2015)

Certains distributeurs indépendants sont conscients du problème d'acheminement des produits qui les rend moins compétitifs. Mais ils n'ont ni les compétences ni les ressources nécessaires pour accéder à d'autres solutions. 
«C'est ma principale problématique : le coût de l'approvisionnement!» (Commerçant indépendant, compte rendu de réunion, 8/11/2016)

\section{Discussion}

\section{La logistique : enjeu majeur}

À la lecture du cas CapLog, nous pouvons considérer que la ruralité du territoire met en exergue certaines problématiques récurrentes des canaux de distribution.

D'abord, la position du consommateur dans la mise en place de canaux de distribution alimentaire paraît ambiguë. D'une part, le consommateur réclame une alimentation plus responsable (Moati, 2018), mais il privilégie aussi la «facilité », souhaitant souvent accéder rapidement à ses achats déjà massifiés, c'est-à-dire regroupés au même endroit afin de limiter ses déplacements (Vaillant et al., 2017). D' autres consommateurs privilégieront au contraire certains modes de distribution en accord avec leurs principes, quitte à assumer des coûts d'achat, de déplacement supplémentaires (Vaillant et al., 2017), et même certaines tâches logistiques (cueillette sur site, emballage des produits...).

Ensuite, l'existence sur le territoire d'acteurs de la distribution, suffisamment motivés pour se développer, semble un prérequis à l'adaptation des canaux. Mais, à l'échelle d'un territoire rural, les canaux de distribution sont épars, ce qui complexifie la logistique des producteurs. Ceux-ci doivent alors envoyer souvent de petites quantités à de nombreux destinataires, ce qui complexifie leur gestion des transports, des stocks et qui leur fait perdre du temps. Pour ces raisons liées à leur cœur de métier, beaucoup de producteurs refusent de s'impliquer dans la distribution locale. Inversement, certains producteurs intègrent les fonctions d'expédition et de distribution, mais regrettent l'aspect chronophage de certaines tâches qui présentent peu de valeur ajoutée ou soulignent l'impact négatif de l'augmentation du prix du pétrole. Quoi qu'il en soit, le pilotage logistique, notamment à travers des mécanismes de mutualisation, représente un enjeu clé pour faire émerger un leader reconnu.

Par ailleurs, l'analyse des suprastructures et infrastructures logistiques apparaît comme un élément majeur de la compréhension des problématiques de distribution en contexte rural. Une disparité d'accès importante au sein d'un territoire et l'augmentation des coûts des carburants aggravent les difficultés logistiques. Cette problématique reste peu étudiée dans les recherches en gestion qui s'intéressent davantage à celles des milieux urbains, notamment lorsqu'elles sont relatives au «dernier kilomètre » (Chanut et Paché, 2013).

Enfin, plus largement, les problématiques de distribution alimentaire locale représentent un levier pour la résilience des territoires (Noireaux, 2017). Dans des régions rurales isolées, elles peuvent jouer un rôle pour résister à la réduction de l'activité économique, à la désertification, à la disparition des services.

\section{Le rôle du leader : une difficile équation}

L'évolution de la demande vers une certaine proximité induit une adaptation des rôles au sein du canal. Si, en milieu urbain, différents leaders, historiques ou non-grossistes, prestataires de services logistiques, grande distribution -, initient déjà des solutions (Libeskind, 2015), en territoire rural, la coordination des flux engendre davantage de difficultés (rentabilité, normalisation...), même si de nombreuses expérimentations existent. Cellesci sont observées au-delà du territoire du Massif central, comme le montre la plateforme Terroirs Ariège Pyrénées, non rentable après cinq ans d'activités (Charvillat, 2017). Dans un contexte rural, le leader 
doit donc avoir une grande connaissance du territoire pour pouvoir adapter au mieux son offre aux spécificités locales (Pouzenc, 2012).

Nos résultats montrent qu'il n'existe pas, dans les territoires ruraux, de leaders «naturels », en raison de l'insuffisance de compétences ou de volonté. Ces deux freins ont déjà été relevés dans la littérature (Stern et El Ansary, 1988 ; Noireaux et Poirel, 2014). Néanmoins, étant donné l'enjeu, quelques acteurs pourraient, en développant un des deux attributs, endosser ce rôle.

Le premier acteur identifié pour piloter les canaux d'alimentation en milieu rural est la grande distribution, qui souhaiterait consolider dans ce domaine son leadership (Pouzenc, 2012). La grande distribution a déjà mis en place des groupes de travail et de pratique ${ }^{2}$. Mais les difficultés persistent du fait des normes logistiques imposées et de la mauvaise connaissance des acteurs des territoires, même si, en milieu rural, certains dirigeants sont intégrés dans le tissu local (Pouzenc, 2012). Inversement, certains producteurs refusent de vendre à la grande distribution pour des raisons éthiques, économiques ou de gestion de la dépendance.

Le deuxième type de leader possible pourrait être les grossistes locaux. Pour cela, encore faut-il qu'ils possèdent la capacité financière d'investir dans des ressources spécifiques (comme des camions multi-compartimentés permettant de transporter des produits frais, secs et surgelés).

2. Table ronde, organisée par LSA et le Sial le 28 juin 2012 sur la politique des grands distributeurs en matière de produits locaux (https:// www.lsa-conso.fr/la-distribution-s-active-sur-lesproduits-locaux,131785), $5 \%$ environ d'augmentation du sourcing pour les TPE et pour les PME en 2014 (http://www.toute-la-franchise.com/viede-la-franchise-A22571-la-grande-distributionaime-les-pro.html) ou encore groupe Proxi du groupe Carrefour.
Or, dans le groupe CapLog, certains d'entre eux ont été extrêmement affaiblis financièrement par la grande distribution. En effet, le développement logistique des distributeurs conduit à diminuer les flux passant par les grossistes indépendants. Cette diminution d'activité impacte leur chiffre d'affaires, mais également leur marge, car les flux transportés sont moindres, la mutualisation difficile (du fait de la diminution des volumes) et la pression sur les prix est forte. La baisse de leur rentabilité les freine dans leur capacité d'investissement. Cet affaiblissement a déjà été relevé dans la littérature (Michel et Pardo, 2012). D'autres semblent satisfaits de leurs activités, mais ne se placent pas comme leader pour des raisons de rentabilité à court terme et de cœur de métier.

Le troisième type de leader possible sont les prestataires de services logistiques. Ces derniers disposent d'une maîtrise de la conception des chaînes logistiques et sont en mesure de fournir des moyens internes ou externes en recourant à d'autres prestataires (Fabbe-Costes et al., 2011). Dans notre étude, aucun prestataire ne souhaitait prendre la direction du canal, trouvant l'opération trop complexe à mettre en place au regard de la rentabilité perçue. Ils ne considèrent pas la distribution en territoire rural comme une cible stratégique ou n'ont pas besoin de ces entreprises pour réaliser leur activité. Ces deux points sont en accord avec la littérature, où l'absence de vision stratégique ou le manque de facilité (au sens strict) sont considérés comme des barrières à la mise en place d'innovations durables par les prestataires de services logistiques (Centobelli et al., 2017).

Les institutions (collectivités locales, chambres consulaires...) apparaissent également comme un type de leader potentiel. En effet, les enjeux de la distribution sont importants pour elles, malgré leur manque de compétences. Néanmoins, elles possèdent une maîtrise de l'aspect contractuel 
des marchés (eau, déchets...). Elles pourraient alors organiser le marché en proposant un appel d'offre spécifique aux prestataires de services logistiques par exemple. Mais se pose alors la question du périmètre de leur activité. En effet, l'organisation de la distribution de produits locaux relève-telle des institutions? Elles peuvent également participer au développement de solutions en proposant des aides financières lors de la mise en place d'un projet privé et/ ou collectif (mise à disposition d'un local, aide à l'investissement des grossistes...). C'est la solution la plus envisagée à court terme au sein de CapLog.

Enfin, une dernière catégorie d'acteurs ne possède aucun des deux attributs de compétence et de volonté requis pour devenir leader logistique. Pour les agriculteurs, les industries de transformation et les distributeurs indépendants, l'accès au rôle de leader paraît difficile. Il faut à la fois qu'ils souhaitent endosser ce rôle, mais également qu'ils développent des ressources et des compétences spécifiques. Si certains contre-exemples existent, notamment celui des AMAP où une association de consommateurs organise le canal, ces initiatives possèdent un certain nombre de limites (Sirieix et Borgne, 2017), liées en particulier à une motivation forte et un degré d'engagement particulièrement élevé des consommateurs impliqués (Oliviet et Coquart, 2010).

Enfin, il serait également possible qu'émerge une forme plus collective de leadership, comme décrit par Noireaux et Poirel $(2014,2017)$, où une solution émane des échanges entre les différentes parties prenantes. Dans ce cas, un tel pilotage permettrait de dépasser certaines limites liées à l'individualité des acteurs, comme le manque de ressources, de compétences (notamment logistique) ou la contrainte d'un acteur trop puissant. Même si cette solution semble séduisante sur le papier, les difficultés de mise en place et de développement de ces stratégies sont nombreuses : difficultés de gestion (lenteur des décisions, communication...), conflits interpersonnels, absence de certaines parties prenantes clés dans le projet, absence de vision commune... (Dari et Paché, 2015). Néanmoins, lorsqu'elles sont appliquées de manière cohérente, elles peuvent se révéler très efficaces (Noireaux et Poirel, 2017).

\section{$*$ \\ * *}

L'étude des canaux de distribution de l'alimentation en milieu rural a permis de faire ressortir leurs spécificités, et plus particulièrement leurs freins à travers les difficultés du leader à développer les trois attributs essentiels à la construction d'un canal fonctionnel.

Tout d'abord, le prestataire ou le grossiste, parfois les mieux placés, se révèlent paradoxalement défaillants. Bien que possédant les compétences nécessaires dont ils usent par ailleurs, ils n'expriment pas forcément de volonté réelle de structurer les énergies afin de définir et d'organiser une solution nouvelle. Par ailleurs, le rôle d'influence du consommateur s'avère une réalité en incitant les institutions administratives et politiques à développer ou à appuyer le développement de solutions. D'abord, parce que des acteurs comme les transporteurs, les grossistes ou les logisticiens ne sauraient généralement investir sans le soutien financier ou matériel des pouvoirs publics. Ensuite, parce que les autorités locales trouvent souvent dans les projets émergents le moyen d'atteindre leurs propres objectifs en matière de redynamisation des territoires.

D'un point de vue managérial, plusieurs pistes d'organisation apparaissent quant à la démarche à mettre en œuvre, au regard des spécificités existantes. Seules des solutions contextualisées et contingentes, négociées par les acteurs eux-mêmes, semblent pouvoir être couronnées de succès. Le rôle 
des institutions administratives et politiques demeure alors important.

Ces recommandations doivent être étayées par des travaux complémentaires. Notre étude est avant tout qualitative et exploratoire et ne concerne que la distribution en zone rurale de moyenne montagne. De nombreuses pistes de recherches découlent de ce travail. La première serait d'approfondir au sein des AMAP les notions de pouvoir, notamment la légitimité perçue de l'association de consommateurs comme leader. Nous avons relevé cette difficulté, mais il s'agit de voir si ce cas relève d'un cas particulier ou pas. La seconde concerne l'étude des stratégies de résistance des acteurs majeurs actuellement dans la distribution rurale. Ainsi, nous avons souligné que les prestataires et certains grossistes ne se sentaient pas légitimes pour développer ce marché. Nous pouvons nous poser la question de leur résistance au changement dans un contexte qui leur est bénéfique. Enfin, l'étude d'une solution collective, mise en place par les acteurs eux-mêmes, accompagnée par une véritable expertise logistique, permettrait peut-être de dégager d'autres solutions. 


\section{RÉFÉRENCES BIBLIOGRAPHIQUES}

Anand P., Stern L. W. (1985). A sociopsychological explanation for why marketing channel members relinquish control. Journal of Marketing Research, vol. 22, $\mathrm{n}^{\circ} 4$, pp. 365-376.

Baumard P., Ibert J. (2007). Quelles approches avec quelles données? In Thiétart R. A. Méthodes de recherche en management, $3^{\mathrm{e}}$ éd., Paris, Dunod, pp. 84-106.

Blézat Consulting, CREDOC, Deloitte (2017). Étude prospective sur les comportements alimentaires de demain et élaboration d'un dispositif de suivi des principales tendances de consommation à destination des entreprises de la filière alimentaire. En ligne: https://agriculture.gouv.fr/ etude-prospective-sur-les-comportementsalimentaires-de-demain.

Cara S., Fournier A., Gaigné C. (2017). Local Food, Urbanization, and Transport Related Greenhouse Gas Emissions. Journal of Regional Science, vol. 57, $\mathrm{n}^{\circ}$ 1, pp. 75-108.

Centobelli P., Cerchione R., Esposito E. (2017). Environmental sustainability in the service industry of transportation and logistics service providers: Systematic literature review and research directions. Transportation Research, Part D: Transport and Environment, $n^{\circ}$ 53, pp. 454-470.

Cerdan C., Boucher F., Sautier D., Fournier S. (2017). Chapitre 21. Les systèmes agroalimentaires localisés. In Caron P. (dir.), Des territoires vivants pour transformer le monde, Versailles, Quae, pp. 136-140.

Chanut O., Paché G. (2013). La culture de mutualisation du PSL peut-elle favoriser l'émergence d'une logistique urbaine durable ? RIMHE, n ${ }^{\circ} 3$, pp. 94-94.

Charvillat E. (2017). Étude des conditions technico-économiques sur le territoire de Tulle agglo pour la création d'une plateforme d'approvisionnement de la restauration collective en produits issus de l'agriculture locale. Mémoire de fin d'études d'ingénieur. Vetagrosup.

Christopher M. (2016). Logistics \& supply chain management. Harlow, Pearson, 275 p.
Converse P. (1958). The other half of marketing. In Seyle A. (ed.), Marketing in transition, Harper and Row, New York, pp. 114-121.

Dari L., Paché G. (2015). Acteurs tiers et stratégies collectives au sein des filières. Le cas du liège en Corse. Économie rurale, $\mathrm{n}^{\circ} 5$, pp. 101-123.

Delavigne A. E. (2001). Aliments purs, aliments saints, la dimension identitaire des peurs alimentaires au Danemark. Anthropology of food. En ligne : https://journals.openedition. org/aof/1052.

Duru M., Justes E., Falconnier G., Journet E. P., Triboulet P., Magrini M. B. (2017). Analyse du concept de santé globale pour accompagner les transitions agricoles et alimentaires : application au cas des légumineuses. Agronomie, Environnement et Sociétés, vol. 7, n 1 , pp. 83-95.

Fabbe-Costes N., Roussat C. (2011). Supply chain integration: views from a Logistics Service Provider. In Supply Chain Forum: an International Journal, vol. 12, $\mathrm{n}^{\circ} 2$, pp. 20-30.

FAO (2010). Definition of sustainable diets. International scientific symposium "Biodiversity and sustainable dietsUnited against hunger. Rome, FAO, 3-5 November 2010. En ligne : http://www.fao. org/3/a-i3004e.pdf.

Gattorna J. L., Tang M. (2003). Formulating a supply chain vision. Gower Handbook of Supply Chain Management, pp. 11-25.

Gontard N., Guillard V., Gaucel S., Guillaume C. (2017). L'emballage alimentaire et l'innovation écologique dans toutes leurs dimensions. Innovations agronomiques, ${ }^{\circ} 58$, pp. 1-9.

Guérin F., Lambert R. (2012). Une rénovation de la gouvernance des chaînes logistiques. Revue française de gestion, vol. 227, $\mathrm{n}^{\circ} 8$, pp. 31-44.

Hlady-Rispal M. (2015). Une stratégie de recherche en gestion-L'étude de cas. Revue française de gestion, vol. $41, \mathrm{n}^{\circ} 253$, pp. 251-266. 
Hunt S. D. (2015). The bases of power approach to channel relationships: has marketing's scholarship been misguided? Journal of Marketing Management, vol. 31, $\mathrm{n}^{\circ}$ 7-8, pp. 747-764.

Ipsos Enquête, Les Français et le consommer local, février 2014. En ligne: https:// www.dijon.fr/content/.../Ipsos_Francais_ ConsommerLocal_fevrier_2014.pdf.

Le Roy F. (2008). The rise and fall of collective strategies. International Journal of Entrepreneurship \& Small Business, vol. 5, $\mathrm{n}^{\circ} 2$, pp. 127-142.

Libeskind J. (2015). Logistique urbaine. Les nouveaux modes de consommation et de livraison. Limoges, FYP, 192 p.

Mallen B. (1964). Conflict and cooperation in marketing channels. In Smith M. L. G. (ed.), Reflections on Progress. Chicago, IL: American Marketing Association.

McCammon Jr B. C. (1970). Perspectives for distribution programming. Vertical marketing systems, $\mathrm{n}^{\circ} 1$, pp. 32-51.

Mehta R., Larsen T., Rosenbloom B. (1996). The influence of leadership style on co operation in channels of distribution. International Journal of Physical Distribution \& Logistics Management, vol. 26, $\mathrm{n}^{\circ}$ 6, pp. 32-59.

Merle A., Piotrowski M. (2012). Consommer des produits alimentaires locaux : comment et pourquoi ? Décisions marketing, $\mathrm{n}^{\circ} 67$, pp. 37-48.

Michel S., Pardo C. (2012). La spécificité du commerce BtoB: quelques repères historiques. Management \& Avenir, $\mathrm{n}^{\circ} 51$, pp. 156-166.

Moati P. (2018). (Dé)penser la consommation : peut-il y avoir une "bonne consommation »? Caen, Éditions EMS.

Muchnik J., Requier-Desjardins D., Sautier D., Touzard J.-M (2007). Systèmes agroalimentaires localisés. Économies et Sociétés, $\mathrm{n}^{\circ} 29$, pp. 1465-1484.

Noireaux V. (2017). Rôle des supply chains locales dans la résilience des territoires: l'exemple des produits alimentaires en Auvergne. Logistique et Management, vol. $25, \mathrm{n}^{\circ} 3$, pp. 225-237.
Noireaux V., Poirel C. (2009). Pouvoir et leadership dans les canaux de distribution. Revue Management et Avenir, $\mathrm{n}^{\circ} 24$, pp. 32-48.

Noireaux V., Poirel C. (2014). Contre-pouvoir et stratégies collectives. Comment contrer le pouvoir d'un puissant partenaire dans le canal de distribution? Revue française de gestion, vol. 338, n 4551, pp. 109-124.

Noireaux V., Poirel C. (2017). Environnement et processus d'adaptation des chaînes logistiques. In Paché G. (dir.), Images de la logistique : éclairages managériaux et sociétaux, Presses universitaires d'Aix-Marseille, pp. 69-75.

Perotti S., Zorzini M., Cagno E., Micheli G. J. (2012). Green supply chain practices and company performance: the case of 3PLs in Italy. International Journal of Physical Distribution \& Logistics Management, vol. 42, n 7, pp. 640-672.

Pouzenc M. (2012). Les grandes surfaces alimentaires contre le territoire... tout contre. Pour, vol. 215-216, n 3, pp. 255-261.

Rose W. J., Bell J. E., $\quad$ Autry C. W., Cherry C. R. (2017). Urban Logistics: Establishing Key Concepts and Building a Conceptual Framework for Future Research. Transportation Journal, vol. 56, $\mathrm{n}^{\circ} 4$, pp. 357-394.

Sirieix L., Le Borgne G. (2017). Vers de nouveaux modèles de consommation? In Lubello, P., Falque, A., Temri, L. (dir.), Systèmes agroalimentaires en transition. Versailles, Quae, coll. «Update Sciences \& Technologies », pp. 89-100.

Stern L. W., El-Ansary A. I. (1988). Marketing Channels. Englewood Cliffs, NJ, Prentice Hall, $621 \mathrm{p}$.

Tang M., Thomas G. (2017). 4 Operating strategy: configuring segmented supply chains. In Gattorna, J. (ed.), Gower Handbook of Supply Chain Management, London, Routledge, pp. 54-67.

Vaillant L., Gonçalves A., Raton G., Blanquart C. (2017). Transport et logistique des circuits courts alimentaires de proximité : la diversité des trajectoires d'innovation. Innovations, vol. 54, n 3 , pp. 123-147. 
Vázquez A. M. (2005). Quality certification, institutions and innovation in local agro-food systems: protected designations of origin of olive oil in Spain. Journal of Rural Studies, vol. $21, \mathrm{n}^{\circ} 4$, pp. $475-486$.
White D. D. Bednar D. A. (1991). Organizational Behavior: Understanding and Managing People at Work. 2nd ed., Boston, MA, Allyn \& Bacon, 655 p.

\section{Annexe I. Acteurs actifs du projet Caplog}

\author{
- Production \\ - Coop de France Rhône-Alpes Auvergne \\ - Chambre régionale d'Agriculture Auvergne \\ - Transformation \\ - Union Régionale des Industries Agro-Alimentaires \\ de l'Auvergne (URIAA Auvergne) \\ - Distribution, transport-logistique \\ - CODI France (Grossiste Allier) \\ - MAGNE Distribution (Grossiste Lozère) \\ - SODICAL-ALLAYRANGUE (Grossiste Cantal) \\ - Groupe La Poste (Auvergne) \\ - Magasin Panier Sympa (Cantal) \\ - MonDriveLocal \\ - SNCF Auvergne Bourgogne Ouest \\ - Fédération du Commerce et de la distribution \\ - Carrefour \\ - Auvergne Bio Distribution (Grossiste Bio) \\ - Collectivités territoriales \\ - Conseil général de la Lozère \\ - Conseil général du Cantal \\ - Conseil général 63 \\ - Association des Inter parcs du Massif central (IPAMAC) \\ - Parc naturel régional (PNR) des Volcans d'Auvergne \\ - Parc naturel régional (PNR) Millevaches en Limousin \\ - Conseil Régional
}

\section{- Structures d'accompagnement}

- Chambre de commerce et d'industrie (CCl)

Auvergne

- Chambre de commerce et d'industrie (CCl)

Lozère

- Chambre de Métiers et de l'Artisanat -

Auvergne

- Caisse des Dépôts et Consignations (Paris)

- ISBA Conseil (Consultant DD)

- Macéo (Association)

- Chambre Régionale de l'Agriculture

- Services de l'État

- Directions régionales des entreprises, de la concurrence, de la consommation, du travail et de l'emploi (DIRECCTE) Auvergne

- Direction Régionale de l'Environnement, de l'Aménagement et du Logement (DREAL) Auvergne (Observatoire des Transports)

- Commissariat général à l'égalité des territoires (CGET) Massif central

- Réseau Rural Auvergne

- Chercheurs

- AgroParisTech

- Université d'Auvergne

- VetAgro Sup 